\title{
Serratia marcesens as a Pathogen in a Case of Diabetic Foot: A Case Report
}

\author{
K. Sharanya*, K. Lakshmi and S. Chitralekha \\ Department of Microbiology, Sree Balaji Medical College and Hospital (Bharath University), \\ Chennai, Tamil Nadu, India \\ *Corresponding author
}

\section{A B S T R A C T}

\section{Keywords}

Serratia

marcescens,

Diabetes mellitus,

Soft tissue infection

Article Info

Accepted:

20 January 2018

Available Online:

10 February 2018
Serratia marcescens causing a soft tissue infection is very rare. But, among the immunocompromised patients it is becoming an important pathogen causing infection. Here we report a case of an elderly male with type 2 diabetes mellitus presenting with severe soft tissue infection due to $S$. marcescens. The diagnosis and antibiotic susceptibility pattern are discussed.

\section{Introduction}

A 61 year old male with history of Type 2 diabetes mellitus for the past 6 years with irregular treatment was admitted with a oneweek history of worsening lower right limb pain, ulcer with discharge of pus, erythema and reduced mobility. The patient was initially stable on admission but rapidly deteriorated. $\mathrm{He}$ was febrile at $38^{\circ} \mathrm{C}$ and blood pressure dropped to $100 / 50 \mathrm{mmHg}$. He was tachycardic and tachypnoeic. His right foot was ulcerated, warm and tender with significant swelling in the right leg.

Blood examination results revealed Serum urea $25 \mathrm{mg} / \mathrm{dl}$, Serum creatinine $0.9 \mathrm{mg} / \mathrm{dl}$, total white cell count of 12300 cells/cumm, platelet count of 3.56 lakhs/cumm, C-reactive protein $192 \mathrm{mg} / \mathrm{L}$, ESR of 100mm.Blood glucose Fasting showed $230 \mathrm{mg} / \mathrm{dl}$ and post prandial showed $316 \mathrm{mg} / \mathrm{dl}$ with HBA1C of $8.32 \%$. Wound debridement was done. Pus was collected and sent to Microbiology laboratory for culture and sensitivity. Patient was started on broad spectrum antibiotics.

Direct Gram stain from the pus showed occasional pus cells and occasional Gramnegative coccobacilli. The pus was inoculated into Nutrient agar,5\% sheep Blood agar, Chocolate agar, Mac-Conkey agar and Thioglycolate broth, After 12 hours of incubation, Nutrient agar showed large red 
moist colonies (Fig. 1), 5\% sheep Blood agar (Fig. 3) and Chocolate agar showed growth of red, opaque moist colonies. Meanwhile MacConkey agar grew Red late lactose fermenting colonies (Fig. 2). Gram staining from colonies was confirmed as Gram-negative cocco bacilli. The Gram-negative bacteria was identified as Serratia marcescens by standard microbiological methods as well as using Vitek 2 Compact along with antibiotic susceptibility pattern.

The organism was found to be susceptible to Cotrimoxazole, Piperacillin+tazobactum, Gentamycin, Tobramycin, Imipenem, Meropenem, Ceftriaxone, Tetracycline, Aztreonam, Chloramphenicol and Resistant to Ciprofloxacin, Cefixime, Ampicillin, Amoxicillin+clavulanate and Cefazolin.

The patient was continued with IV Antibiotics based on the sensitivity report and blood sugars were closely monitored. Inflammatory markers like CRP \& ESR were also monitored regularly. The patient's general condition improved and was shifted to oral antibiotics. Patient was discharged later as his CRP returned to normal.

\section{Results and Discussion}

Serratia marcescens is an emerging opportunistic pathogen which has gained global importance in the recent years. It is commonly recognized as hospital acquired infection. However, it has also been reported that $47 \%$ of Serratia bacteremias could be community acquired (Rehman et al., 2012).

S. marcescens is a gram negative bacteria belonging to the genus Serratia, member of Enterobacteriaceae family. The characteristic feature of this bacterium is its distinctive pigmentation, which was used as a biological marker in the olden days (Gaughran, 1969).
Serratia usually inhabits respiratory and urinary tracts where it may cause opportunistic infections. But the organism has also been implicated in various other infections like osteomyelitis, endocarditis, cellulitis, necrotizing fasciitis, blood stream infections, wound infections, etc (Herra and Falkiner, 2015; Anía, 2015).

Colonisation and infection may be aggravated by hospitalisations, increased usage of antibiotics, etc. In the recent years, Serratia has been increasingly reported as opportunistic pathogen. Various predisposing factors for Serratia bacteremia include surgery, antibiotic usage, steroids, diabetes (Crowder et al., 1971).

Serratia is ubiquitous, found in sewage, soil and water. Predisposing factors for Serratia soft tissue injuries could be skin ulcers, biopsy, vascular injuries compromising the skin integrity, prior surgery, etc (Crowder et al., 1971; Bennett et al., 1995).

In our case, patient has developed cellulitis in his leg.

The risk factors included recent hospitalization, antibiotic usage, and immunocompromised state due to diabetes. Huang et al., reported Serratia marcescens causing necrotizing fasciitis in patients on steroid therapy (Huang et al., 1999).

Brenner et al., reported Serratia cellulitis due to infected intravenous catheter in a patient with bacteremia (Brenner DE and Lookingbill DP, 1977).

Literature reveals that Serratia often produce bullae in patients with soft tissue infections similar to beta hemolytic streptococci and Staphylococcus aureus (Huang, 1999; Bornstein, 1992; Zipper, 1996; Cooper, 1998). 
Int.J.Curr.Microbiol.App.Sci (2018) 7(2): 2293-2297

Fig.1 Nutrient agar showing large red moist colonies

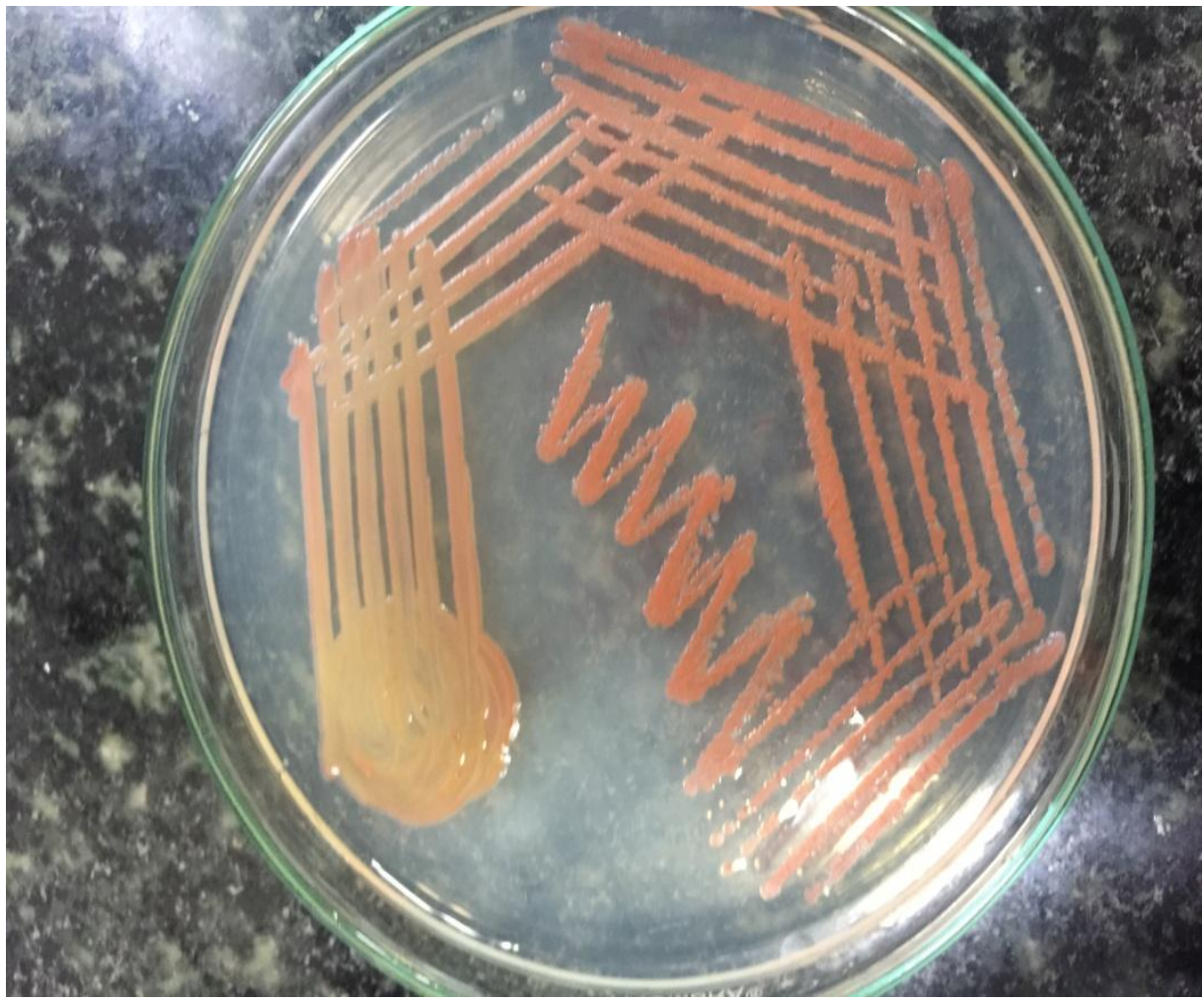

Fig.2 Mac-Conkey agar showing Red late lactose fermenting colonies

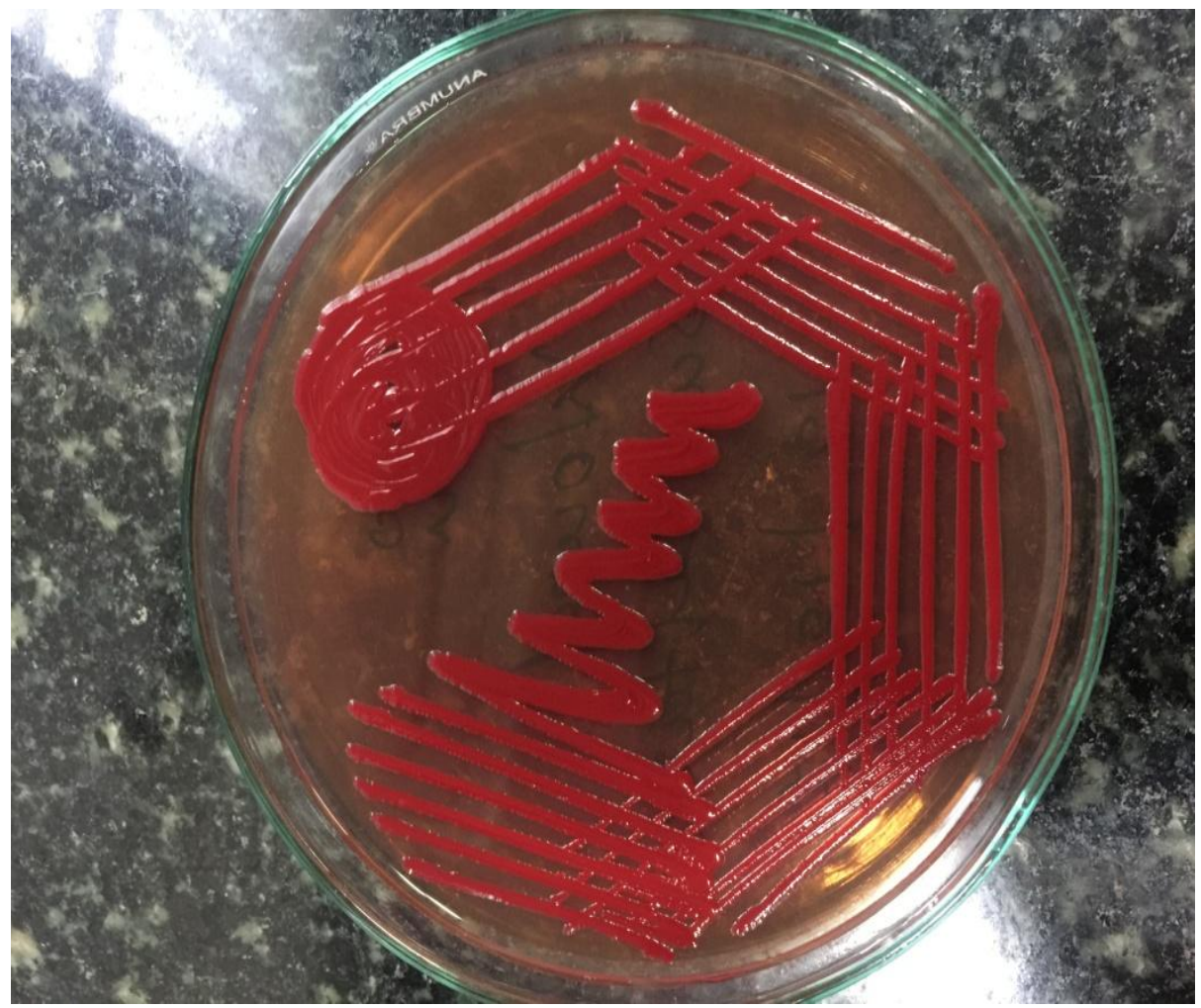


Fig.3 Blood agar showing Red, opaque moist colonies

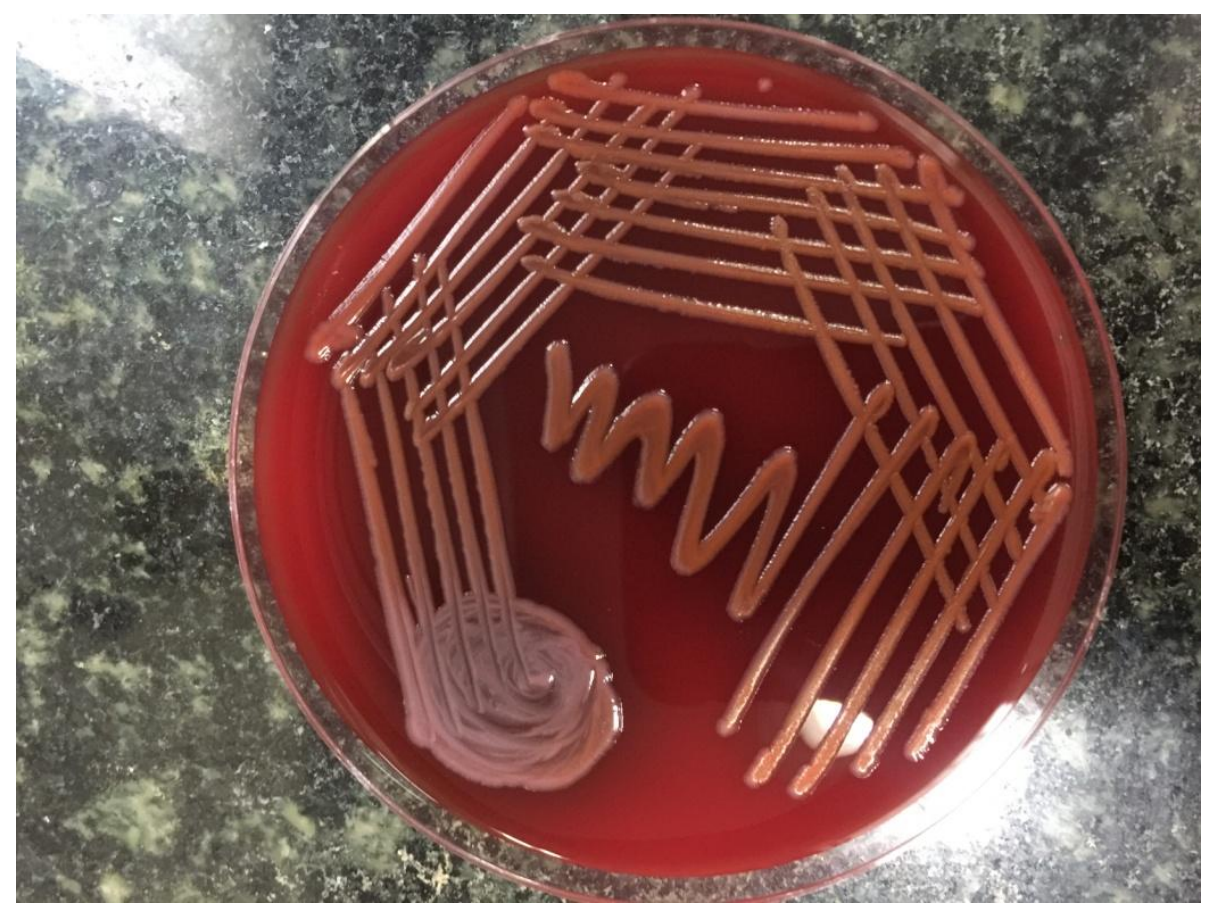

Serratia has been reported rarely from soft tissue infections. In few of the reported cases, diabetes has been found to be the underlying precipitating factor (Zipper, 1996; Kaplan, 1988). Since there are no specific clinical signs and symptoms, the clinicians should be more suspicious to diagnose Serratia in patients with underlying risk factors like diabetes, immunosuppression, etc.

Conventionally, Serratia was diagnosed using Analytical Profile Index (API) 20E (bioMerieux) phenotypic microbial identification system (Hejazi and Falkiner, 1997). With the widespread usage of automated systems, the laboratory diagnosis of Serratia is commonly done with Vitek 2 (bio-Merieux), BD Phoenix (BD Diagnostics, Sparks), etc (Menozzi et al., 2006). Species level identification of Serratia isolates can be done by MALDI-TOF MS (matrix-assisted laser desorbtion/ionization time-of-flight mass spectrometry) which is an automated identification system. The common empirical drug regimen for soft tissue infections is flucloxicillin and benzylpenicillin. However, in cases with suspicion of Serratia, antibiotics with gram negative coverage should also be included. Serratia is commonly resistant to macrolides, ampicillin and first generation cephalosporins (Anía, 2015). Increasing resistance has been reported to gentamicin (Herra and Falkiner, 2015). In our case, the organism has been found to be resistant to cefazolin, but susceptible to gentamicin.

Third generation cephalosporins may be used along with aminoglycosides in treating the cases of Serratia. In this case, the organism was found to be susceptible to ceftriaxone as well as carbapenem group of drugs. Few ESBL strains of Serratiahas been reported to be resistant to third generation cephalosporins (Anía et al., 2015). In such cases, fourth generation cephalosporins or piperacillin tazobactam antibiotics may be tried. Carbapenem group of drugs have also been shown to be effective in resistant cases. However, in carbapenemase producing strains this drug may be ineffective. 
In conclusion, in immunocompromised patients, the cellulitis should be diagnosed and treated with high index of suspicion. Rare pathogens like Serratia require prompt identification and proper management. Further, multidrug resistant strains are becoming common with Serratia infection. Hence, the definitive treatment should be initiated based on antibiotic susceptibility testing. Since the mortality rates are high with Serratia soft tissue infections, early debridement and appropriate antibiotic therapy is the treatment of choice.

\section{References}

Anía BJ. Serratia medication. Cited 8 June 2015. Available at: http://emedicine.medscape.com/article/2 28495-medication\#1.

Bennett SN, McNeil MM, Bland LA, et al., Postoperative infections traced to contamination of an intravenous anesthetic, propofol. $\mathrm{N}$ Engl J Med 1995; 333:147-154.

Bornstein PF, Ditto AM, Noskin GA. Serratia marcescens cellulitis in a patient on hemodialysis. Am J Nephrol 1992; 12:374-376.

Brenner DE, Lookingbill DP. Serratia marcescens cellulitis. Arch Dermatol 1977; 113:1599-1600.

Cooper CL, Wiseman M, Brunham R. Bullous cellulitis caused by Serratia marcescens. Int J Infect Dis 1998; 3:3638.

Crowder JG, Gilkey GH, White AC. Serratia marcescens bacteremia. Arch Intern
Med 1971; 128:247-253.

Gaughran ER. From superstition to science: the history of a bacterium. Trans NY Acad Sci., 1969; 31: 3-24.

Hejazi A, Falkiner FR. Serratia marcescens. J. Med. Microbiol., 1997; 46: 903-912

Herra C, Falkiner FR. Serratia marcescens. Cited 8 June 2015. Available at: http://www.antimicrobe.org/b26.asp.

Huang JW, Fang CT, Hung KY, et al., Necrotizing fasciitis caused by Serratia marcescens in two patients receiving corticosteroid therapy. J Formos Med Assoc 1999; 98: 851-854

Kaplan H, Sehtman L, Ricover N, Slatkis D, Lacentre E. Serratia marcescens: cutaneous involvement. Preliminary report. Med CutanIberoLat Am 1988; 16:305-08.

Menozzi MG, Eigner U, Covan S, Rossi S, Somenzi P, Dettori G, Chezzi C, Fahr AM. Two-center collaborative evaluation of performance of the BD phoenix automated microbiology system for identification and antimicrobial susceptibility testing of gram-negative bacteria. J Clin Microbiol 2006; 44; 4085-4094.

Rehman T, Moore TA, Seoane L. Serratia marcescens necrotizing fasciitis presenting as bilateral breast necrosis. $\mathbf{J}$ Clin Microbiol 2012; 50:3406-08. http://dx.doi.org/10.1128/JCM.0084312

Zipper RP, Bustamante MA, Khatib R. Serratia marcescens: a single pathogen in necrotizing fascii- tis. Clin Infect Dis 1996; 23:648-649.

\section{How to cite this article:}

Sharanya, K., K. Lakshmi and Chitralekha, S. 2018. Serratia marcesens as a Pathogen in a Case of Diabetic Foot: A Case Report. Int.J.Curr.Microbiol.App.Sci. 7(02): 2293-2297. doi: https://doi.org/10.20546/ijcmas.2018.702.277 